\title{
Ellipsometric light scattering to probe the interface of colloids - current applications and future challenges
}

\author{
Andreas Erbe ${ }^{1, a}$ and Reinhard Sigel ${ }^{2}$ \\ 1 Max-Planck-Institut für Eisenforschung GmbH, Max-Planck-Str. 1, 40237 Düsseldorf, Germany \\ 2 University Fribourg, Adolphe Merkle Institute, Chemin du Musée 3, Perolles, CH-1700 Fribourg, Switzerland
}

\begin{abstract}
In recent years, ellipsometric light scattering (ELS) has been developed into a technique which can be used to characterise the interface between spherical colloidal particles and their surrounding medium. Here, we give an overview over previous successful applications of the technique, and its current limits. The successful applications include the characterisation of temperature-dependent swelling of a thermo-sensitive polymer coating on a latex particle, the measurement of birefringence and molecular orientation in a vescile shell, and the characterisation of the ion distribution around electrostatically stabilised latex particles. As a result of the characterisation of the ion distribution, disagreement with the Poisson-Boltzmann description has been reported before. Here, a few more experimental results on latex particles in the presence of $\mathrm{CsBr}$ are discussed.
\end{abstract}

\section{Introduction}

The colloid science has seen many applications over centuries. From the medieval colouring of windows to modern paints and adhesives there are many applications that rely on matter on the length scale below $1 \mu \mathrm{m}$. Furthermore, in nature there are colloids determining the colour of species and structures on the colloidal length scale facilitating mass transport on the intracellular level.

Every time structures containing colloids are assembled, the control over their stability is of crucial importance. Van der Waals attractions, which promote particle aggregation and phase separation from the liquid phase have to be outranged by other, repulsive interactions. Such a stabilization is decided on the interface between the particles and their surrounding medium. Despite its importance, not a lot is know about the structure of these interfaces in solution. Available techniques include routine measurements of electrophoretic mobilities, wide-spread small-angle X-ray and neutron scattering. The structure can be probed by the recently developed and interface sensitive visible-ir sum frequency generation [1-3]. In recent years, we have established ellipsometric light scattering (ELS) as a technique to probe thickness, refractive index, and anisotropy of colloidal interfaces [4-8]. Here, we give an overview of the technique, highlight previous applications from our lab, and show current limits, which can be overcome only with more method development.

\section{Experimental Setup}

The full details of the setup are presented elsewhere [5]. Briefly, based on a commercial setup (ALV GmbH, Ger-

\footnotetext{
a e-mail: a.erbe@mpie.de, aerbe@arcor.de
}

many) for concurrent static ligth scattering (SLS) and dynamic light scattering (DLS), two lasers can be chosen to be focused on a sample through a polarisation state generator, and analysed through a polarisation state analyser, before being detected either by a photomultiplier or an avalanche photodiode. The range of scattering angles $\theta$ is $12^{\circ}$ to $150^{\circ}$.

The polarisation state generator was a combination of a linear polariser, followed by a compensator plate giving $\lambda / 4$ retardation at $\lambda=633 \mathrm{~nm}-\lambda / 4$ retardation at $\lambda=532 \mathrm{~nm}$, where $\lambda$ is the light wavelength. The polarisation state analyser was a compensator of the same type, followed by a polariser.

A drawback of the first experimental implementation is the long duration of a measurement, which takes typically one night instrument time per wavelength for the required angular range. The involved commercial instrument is optimized for DLS, i.e. the detection of stochastic intensity fluctuations in the scattering signal, caused by the diffusion of the scattering particles. Thus, sufficient time averaging is required in ELS measurements in order to determine the average scattering intensity with sufficient accuracy. For faster measurements in a second experiment, the point detection of the optical fiber was replaced by a two dimensional detection with a CCD camera [9]. A whole range of scattering angles is recorded simultaneously. Futher, different pixels of the camera related to the same scattering angle represent different degrees of rotation of the scattering plane (defined by the directions of incident and scattered light) towards the fixed polarization coordinates of the incident beam. Therefore, each picture contains several polarization settings. The larger area of the CCD chip collects more light, thus increasing the sensitivity. With all these improvements, a set of ELS measurements at $7 \lambda$ based on two lasers and a mercury lamp was collected within 5

This is an Open Access article distributed under the terms of the Creative Commons Attribution-Noncommercial License 3.0, which permits unrestricted use, distribution, and reproduction in any noncommercial medium, provided the original work is properly cited. 
hours total measurement time [9]. Details of the optimized apparatus and the measurements at $7 \lambda$ will be the subject of a separate publication.

\section{Polarisation analysis}

With polariser and compensator before and compensator and analyser after the sample, a synthesis and analysis of any arbitrary state of polarisation is possible, hence all elements of the Mueller matrix can be measured simply by intensity measurements. Experimentally, we are interested in the determination of the Mueller matrix elements of the scattering samples. So far, we have focused on spherical particles, which simplifies the Mueller matrix. For a single spherical particle, all nonvanishing Mueller matrix elements can be written in terms of the two nonvanishing amplitude scattering coefficients $S_{1}$ and $S_{2}$ [10]. Their relation to the Mueller matrix elements $M_{l m}$ is given as [10]

$$
\begin{aligned}
M_{11} & =\frac{1}{2}\left(\left|S_{2}\right|^{2}+\left|S_{1}\right|^{2}\right) \\
M_{12} & =\frac{1}{2}\left(\left|S_{2}\right|^{2}-\left|S_{1}\right|^{2}\right) \\
M_{33} & =\frac{1}{2}\left(S_{2}^{*} S_{1}+S_{2} S_{1}^{*}\right) \\
M_{43} & =\frac{i}{2}\left(S_{2}^{*} S_{1}-S_{2} S_{1}^{*}\right) .
\end{aligned}
$$

Furthermore, $M_{22}=M_{11}, M_{44}=M_{33}, M_{21}=M_{12}, M_{34}=$ $-M_{43}$, and all other $M_{l m}=0$.

The amplitude scattering coefficients $S_{1}$ and $S_{2}$ are the diagonal elements of the Jones matrix of a spherical particle,

$$
\left(\begin{array}{c}
E_{H} \\
E_{V}
\end{array}\right)^{(f)}=\frac{\exp [i k(d-z)]}{-i k d}\left(\begin{array}{cc}
S_{2} & 0 \\
0 & S_{1}
\end{array}\right)\left(\begin{array}{c}
E_{H} \\
E_{V}
\end{array}\right)^{(i)}
$$

where $\left(\begin{array}{c}E_{H} \\ E_{V}\end{array}\right)$ defines a polarisation with basis states parallel $\left(E_{H}\right)$ and perpendicular $\left(E_{V}\right)$ to the scattering plane (for a horizontally orientated scattering plane these basis polarizations are horizontal and vertical). The index $f$ denotes the final (scattered) state, while $i$ is used to designate the initial (incident) state. The wave vector of the incoming light is $k, z$ is the position of the scatterer along the beam and $d$ is the distance between scatterer and detector.

For polydisperse samples, and for illumination with anything other than a plane wave, the situation becomes more involved. In these cases, one needs to consider the exact composition of the scattering matrix, or the respective Mueller matrix. In turn, a proper way of parallel decomposition would be needed to retrieve details about the polydispersity [11]. How the different species contribute to the overall signal, i.e. how exactly the averaging is performed is a function of the measurement scheme, as discussed previously [4].

Different measurement schemes of the Mueller matrix elements based on intensity measurements with different polarisations have been described and realised [10]. Such intensity-based schemes fall into the simplest type of polarimetric experiments, and have their limits in accuracy, especially if low intensities need to be measured. In ELS, instead of focusing on the measurement of all matrix elements over a large angular range, we exploit symmetry considerations to measure few meaningful parameters with high accuracy and high angular resolution in an angular range with contains the information about the interface of particles.

The details of the ellipsometric measurement scheme employed here have been described elsewhere $[4,5]$. In a nutshell, the intensity $I$ is measured at different polariser $P$ and analyser $A$ angles at a fixed compensator angle, and subjected to a fitting procedure of the equation

$$
\begin{aligned}
I(P, A)= & I_{d}+\left\langle\left|S_{1}\right|^{2}\right\rangle+\left\langle\left|S_{2}\right|^{2}\right\rangle+I_{s}\left\{1+\cos ^{2}(\theta)\right\} \\
& -\cos \left(2 A+2 \delta_{A}\right) \\
& {\left[\left\langle\left|S_{1}\right|^{2}\right\rangle-\left\langle\left|S_{2}\right|^{2}\right\rangle+I_{s}\left\{1-\cos ^{2}(\theta)\right\}\right] } \\
& +\sin \left(2 A+2 \delta_{A}\right) \\
& {\left[\left\langle\left\langle\left. S_{1}\right|^{2}\right\rangle-\left\langle\left|S_{2}\right|^{2}\right\rangle\right\} \tan \left(2 \Psi_{\mathrm{Q}}\right) \sin (2 P-\Delta)\right] } \\
+ & \sin \left(2 A+2 \delta_{A}\right) 2 I_{s}|\cos (\theta)| \sin (2 P),
\end{aligned}
$$

where $\delta_{A}$ is (formally) the adjustment error in the analyser, $I_{d}$ the detector dark signal, and $I_{s}$ the solvent's scattering intensity detected with vertical polarisation. This procedure is not optimized for speed but for accuracy. It corresponds to the two-zone-averaging of classical reflection ellipsometry, where most imperfections of the optical components cancel out in first order [12]. From the fitting results, the experimental quantities $\tan \left(\Psi_{\mathrm{Q}}\right)$, with

$$
\begin{aligned}
\tan \left(2 \Psi_{Q}\right) & =\frac{2\left|\left\langle S_{1}^{*} S_{2}\right\rangle\right|}{\left\langle\left|S_{1}\right|^{2}\right\rangle-\left\langle\left|S_{2}\right|^{2}\right\rangle}, \\
\tan \left(\Psi_{I}\right) & =\sqrt{\frac{\left\langle\left|S_{2}\right|^{2}\right\rangle}{\left\langle\left|S_{1}\right|^{2}\right\rangle}}, \text { and } \\
\Delta= & \arg \left(\left\langle S_{1}^{*} S_{2}\right\rangle\right)
\end{aligned}
$$

are extracted $[4,5]$. The angular brakets denote averaging over particles with different sizes in a polydisperse mixture, over particles at different positions a beam deviating from a plane wave, and over different wavelengths if quasimonochromatic light is used. The two different quantities $\tan \left(\Psi_{\mathrm{Q}}\right)$ and $\tan \left(\Psi_{\mathrm{I}}\right)$, are two versions of the ellipsometric amplitude ratio $\tan (\Psi)$, which contain the same scattering matrix elements, i.e. the same Mueller matrix elements. For monodisperse samples under illumination with a monochromatic plane wave, $\tan \left(\Psi_{\mathrm{Q}}\right)=\tan \left(\Psi_{\mathrm{I}}\right)$. While the intensity-based quantity $\tan \left(\Psi_{\mathrm{I}}\right)$, which can also be derived from polarised static light scattering as the simplest possible intensity-based polarimetric experiment, contains coherent and incoherent scattering contributions, the quantity $\tan \left(\Psi_{\mathrm{Q}}\right)$ filters the coherent scattering contributions only $[4,5]$.

In order to model the experimental quantities, $S_{1}$ and $S_{2}$ are calculated using Mie theory. Within the framework of this theory, both $S_{1}$ and $S_{2}$ are expressed as an infinite 
series of products of the Mie coefficients $a_{l}$ and $b_{l}$ and Legendre polynomials. The number of $a_{l}$ and $b_{l}$ which need to be included in the series is a function of the size of particles, and increases with increasing size [10].

\section{Examples}

The reason for the adoption of the measurement scheme becomes clear when considering a point scattering centre and the analogy between light scattering from a spherical particle and reflection from a planar interface [6]. In reflection ellipsometry, the sensitivity to deviations from the ambient-substrate system can be detected with high sensitivity for transparent samples at the Brewster angle. For ELS, there is also such a Brewster angle, around the scattering angle of $90^{\circ}$. While in reflection ellipsometry, the Brewster angle is mainly governed by the minimum of the amplitude reflection coefficients with p-polarisation, it corresponds to the minimum in $S_{2}$ in ELS. The special sensitivity of measurements to deviations from the ideal point scattering behaviour especially due to anisotropy near that minimum was noticed before [13], but is only now exploited on a regular basis.

This Brewster angle is illustrated in Figure 1. It compares measurements from sodium dodecyl sulfate (SDS) micelles in salt-free aqueous solution to the expected results for a point scatterer (Rayleigh scattering). There is a clear minimum in both measured the $\tan \left(\Psi_{\mathrm{Q}}\right)$, as well as $\tan \left(\Psi_{\mathrm{I}}\right)$. This minimum goes along with a "jump" in $\Delta$ from $0^{\circ}$ to $\pm 180^{\circ}$. As discussed previously, the minimum in $\tan \left(\Psi_{\mathrm{I}}\right)$ is not as sharp as the minimum in $\tan \left(\Psi_{\mathrm{Q}}\right)[4,5]$. The sharper minimum for $\tan \left(\Psi_{\mathrm{Q}}\right)$ shows the benefits of the ellipsometric detection scheme over the simple intensitybased quantities.

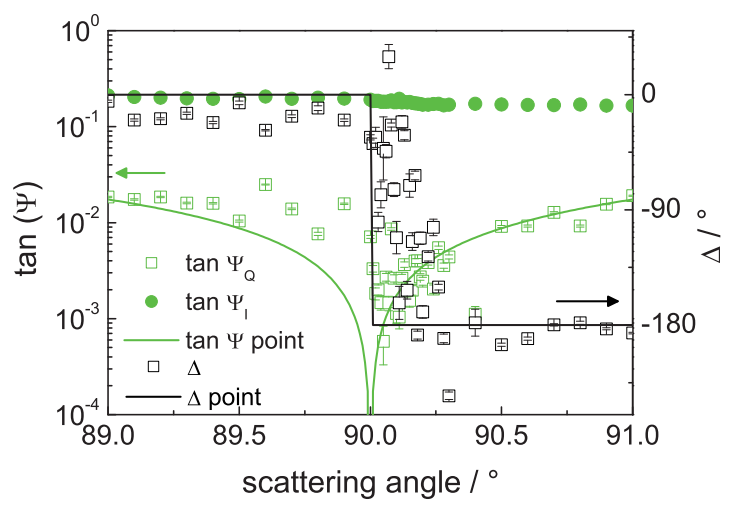

Fig. 1. Ellipsometric parameters $\tan \left(\Psi_{\mathrm{Q}}\right)$ (green open squares), $\tan \left(\Psi_{\mathrm{I}}\right)$ (green circles), and $\Delta$ (black open squares) of SDS micelles, compared to $\tan \left(\Psi_{\mathrm{Q}}\right)$ (green line) and $\Delta$ (black line) for a point scatterer at a wavelength of $532 \mathrm{~nm}$.

The radius of the SDS micelles was determined by dynamic light scattering as $(3.6 \pm 0.5) \mathrm{nm}$. Compared to a wavelength of $532 \mathrm{~nm}$, these micelles are usually considered as point scatterers. A full Mie Mie computation confirms the point scattering behaviour. As Figure 1 shows, the behaviour is indeed very close: the minimum in $\tan \left(\Psi_{\mathrm{Q}}\right)$ (i.e. the Brewster angle) is close to the minimum for the point scatterer. There are, however, remaining deviations which are above the experimental error, in the exact position of the Brewster angle. While the ideal point scatterer shows a Brewster angle of $90^{\circ}$, in the case of SDS we observe a $\sim 0.1^{\circ}$ shift to higher scattering angles. This shift can be caused by ion adsorption, far-reaching changes in the solvent structure around the particle, or anisotropy due to internal structure $[5,7,8]$. The shift shows the high sensitivity of ELS for Mie scattering effects beyond the simple approximation in of Rayleigh scattering.

The position of the Brewster angle reflects the size of the particles. With positive contrast, i.e. if the refractive index of the particle is larger than the the refractive index of the medium, the Brewster angle shifts to larger angles with increasing particle size [5]. Note the high sensitivity of ELS on particle size. In SLS, in contrast, the minimum size for the radius of gyration which can be measured is usually indicated as $\lambda / 20$. If the particles are sufficiently small so that only Mie coefficients $a_{1}, a_{2}, b_{1}$ and $b_{2}$ need to be included in the computation, the position of the Brewster angle $\theta_{B}$ can be computed analytically as [14]

$$
\begin{aligned}
\cos \left(\theta_{B}\right)= & \frac{-3 a_{1}-5 b_{2}+\sqrt{\left(3 a_{1}+5 b_{2}\right)^{2}-40 a_{2}\left(3 b_{1}-5 a_{2}\right)}}{20 a_{2}} .
\end{aligned}
$$

Figure 1 also implies that far away from the Brewster angle, many systems show very similar parameters, an expected phenomenon for particles which are smaller than the wavelength. This similarity is shown for triblock copolymer colloids ([7], see also 4) in Figure 2 over the complete range of scattering angles available. The inset shows in detail the results near the Brewster angle. From such measurements, information about the layers around the particles can be extracted. Far away from the Brewster angle there is no special feature in the curve, if the particles are sufficiently small (see section 6), implying that the ELS curves of particles of many different types look identical. In the region far from the Brewster angle, deviations from the ideal behaviour can be shown to arise from nonidealities in the optical setup. Measurements far away from the Brewster angles have been used to calibrate the birefringence of the sample cells used $[6,5]$.

\section{Applications}

Ellipsometric light scattering has been successfully applied to the characterisation of the temperature dependence of the layer properties of poly $(N$-isopropylacryl amide) (PNIPAM) in poly(ethylene glycol)-block-poly( $N$-isopropylacryl amide)-block-poly(methylmethacrylate) triblock copolymer particles. PNIPAM is known as a thermoresponsive polymer with an lower critical solution temperature $T_{\mathrm{LCST}}$ around $35^{\circ}$. Thus, the polymer is soluble below $T_{\mathrm{LCST}}$ and 


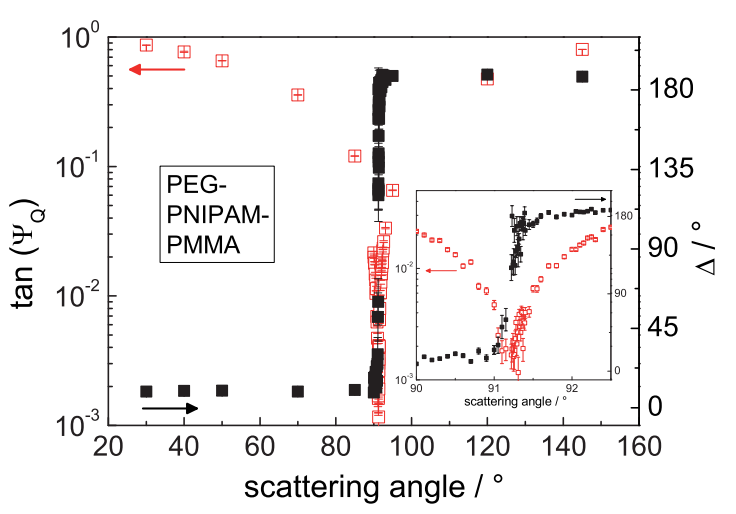

Fig. 2. Ellipsometric parameters $\tan \left(\Psi_{\mathrm{Q}}\right)$ (red open squares) and $\triangle$ (black filled squares) of a dispersion of PEG-PNIPAM-PMMA particles [6] measured at $633 \mathrm{~nm}$ at $25^{\circ} \mathrm{C}$. An inset shows a magnified view of the region around the Brewster angle with the same quantities as the main figure.

insoluble above $T_{\mathrm{LCST}}$. By ELS, it was possible not only to determine the temperature-dependent decrease of the layer thickness of the layers on the particles, but also the temperature-dependent increase in the layer refractive index. For the characterisation of such particles, the index sensitivity is a major advantage over small angle scattering techniques, which have to rely on contrast variation techniques in order to elucidate the contrast of the layers [6].

A further successful application was the characterisation of the ion distribution around charged, spherical latex particles stabilised by perfluoroctanate or SDS [7]. Different concentrations of $\mathrm{NaCl}$ were added, and a pronounced shift in the location of the Brewster angle was observed. The $\mathrm{NaCl}$ concentration was kept well below the critical coagulation concentration, so the repulsive electrostatic interactions remain sufficiently strong to avoid particle aggregation and to keep a situation with isolated particles in solution. For the different $\mathrm{NaCl}$ concentrations, a thickness parameter and a refractive index could be extracted from the measurements. For high concentrations of $\mathrm{NaCl}$, both quantities are inconsistent with the frequently applied Poisson-Boltzmann (PB) description of the ion distribution. The behaviour observed resembles a prewetting transition, and may be due to ion-ion interactions, effects of water structure or van-der-Waals interactions between the low molecular weight ions and the colloidal particles. All the aforementioned effects are not considered in the PB description. This experiment is one of the few studies in the field of the characterisation of the ion distribution about such large particles [7]. A related observation concerncs the wetting of colloidal interfaces in an aqueous medium by oct-1-en [15].

In follow-up experiments, $\mathrm{CsBr}$ was used instead of $\mathrm{NaCl}$ as a salt with higher refractive index contrast. The results of the ellipsometric curves are displayed in Figure 3 . As in the previous results for $\mathrm{NaCl}$, there is also a characteristic shift in the Brewster angle, due to the presence of the $\mathrm{CsBr}$ near the surface. In this case, however, the simple core-shell model employed in previous analyses [7] is not sufficient to describe the data. Comparing the raw data published previously with $\mathrm{NaCl}$ [7] to those obtained with $\mathrm{CsBr}$, there are slight differences. While with addition of $\mathrm{NaCl}$, the Brewster angle shifts to lower scattering angles with increasing salt concentration, the opposite is observed with addition of $\mathrm{CsBr}$. The overall shift in the Brewster angle with increasing salt concentration is lower for $\mathrm{CsBr}$ $\left(0.05^{\circ}\right.$ for $\mathrm{CsBr}, 0.1^{\circ}$ for $\left.\mathrm{NaCl}\right)$. The fact that in the case of the experiments with $\mathrm{CsBr}$, the minimum of $\tan \left(\Psi_{\mathrm{Q}}\right)$ is less pronounced, as well as the slight overall shift of the Brewster angle may be related to an non-perfect alignment of the setup while these experiments where conducted.

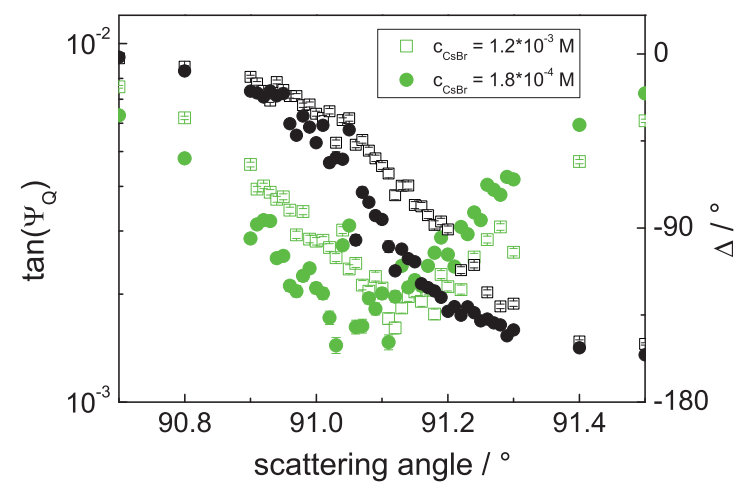

Fig. 3. Ellipsometric parameters $\tan \left(\Psi_{\mathrm{Q}}\right)$ (green) and $\Delta$ (black) at $532 \mathrm{~nm}$ of poly(styrene) latex Eur41 as used in previous experiments [5,7] in the presence of $1.2 \times 10^{-3} \mathrm{M} \mathrm{CsBr}$ (open squares), and $1.8 \times 10^{-4} \mathrm{M} \mathrm{CsBr}$, respectively.

The non-perfect alignment may contribute to the fact that these data cannot quantitatively be fit to the oversimplified model of a single shell with constant refractive index. Some qualitative insight can nevertheless be obtained. Simulations performed previously show that the changes in the signal predicted by the PB description are below the error limit of detection [7]. Here, a significant effect is found for $\mathrm{CsBr}$. Even though the overall refractive index contrast is larger for $\mathrm{CsBr}$, the change in the raw data is smaller than for $\mathrm{NaCl}$, indicating a difference between the salts. It must be stated, however, that enrichment of one ionic species on the surface goes along with depletion of another species, leading to involved effects on the overall refractive index.

A more recent application of ELS is the characterisation of the radial anisotropy, i.e. birefringence, of small particles. In case of a radial birefringence, the spherical symmetry of the particle is kept, which is why it cannot be detected in depolarised light scattering. The first application was the characterisation of the birefringence of a lipid bilayer in a spherical lipid vesicle of birefringence of unilamellar lipid vesicles consisting of 1,2-Dipalmitoyl$s n$-glycero-3-phosphocholine (DPPC) [8]. From the birefringence, with the knowledge of the anisotropy of the po- 
larisability, the tilt angle of the lipid acyl chains with respect to the bilayer normal could be determined. The value was found to be $(29 \pm 0.5)^{\circ}$, which is close to the tilt angle found in planar bilayers. To our knowledge, this experiment was the first successful determination of the tilt angle in a spherical structure.

As a next step, the internal anisotropy of poly(styrene) spherical latex particles could be shown [5]. Such poly(styrene) particles are frequently assumed to be isotropic, but again due to the fact that one deals with radial anisotropy, the anisotropy is difficult to determine by other methods. On the other hand, considering the growth mechanism of a latex particle and the action of the interface tension, an internal anisotropy is not surprising. In fact, if there is an anisotropy, it is expected to be radial, therefore does not show up in many experiments.

\section{Outlook}

In the previous applications, the focus was on spherical particles. Non-spherical, polydisperse particles, especially with an anisotropic internal structure, are important systems, because they are frequently occuring for inorganic colloidal systems. The understanding of such particles presents, however, potentially some difficulties. Sequential decomposition of Mueller matrices may be useful in order to separate the effects in the signals from different origins [11, 16,17].

Previously, especially for large particles, cases where observed where the data at the different wavelengths employed in the study cannot be fit simultaneously to a physically suitable model. The reason for this is unclear so far.

For metal particles, computations have shown considerable deviations of the Brewster angle from $90^{\circ}$ have been found already for very small particle sizes. This behaviour is due to the large refractive index difference. So far, metal particles have not been investigated at all by ELS, due to potential problems with their absorption, and technical issues in the fits. An ELS investigation of metallic particles is, however, still on the agenda.

One other interesting problem to be explored are nonspherical particles. The setup can basically be used also in other measurement schemes. Therefore, Mueller matrix elements from non-spherical particles can also be determined. However, the extraction of meaningful data requires fast and reliable forward calculation of the scattering functions, which is still a sticking point.

Overall, ELS has a high potential to develop over the coming years, from routine sample analysis, e.g. in industrial environments, to high-end scientific problems, such as follow-ups on the ion distribution question, or induced anisotropy.

\section{Acknowledgements}

Most of the experimental work was done at the Max Planck Institute of Colloids and Interfaces in Potsdam. We thank Klaus Tauer for the supply of high-quality samples and
Birgit Schonert for help in sample preparation. Funding by the Max Planck Society and the German Science foundation (Project SI 1092/1-1) is gratefully acknowledged.

\section{References}

1. S. Roke, W.G. Roeterdink, J.E.G.J. Wijnhoven, A.V. Petukhov, A.W. Kleyn, M. Bonn, Phys. Rev. Lett. 91, (2003) 258302

2. S. Roke, M. Bonn, A.V. Petukhov, Phys. Rev. B 70, (2004) 115106

3. S. Roke, O. Berg, J. Buitenhuis, A. van Blaaderen, M. Bonn, Proc. Nat. Acad. Sci. 103, (2006) 13310-13314

4. R. Sigel, A. Erbe, Appl. Opt. 47, (2008) 2161-2170

5. A. Erbe, K. Tauer, R. Sigel, Langmuir 25, (2009) 2703-2710

6. A. Erbe, K. Tauer, R. Sigel, Phys. Rev. E, 73, (2006) 031406

7. A. Erbe, K. Tauer, R. Sigel, Langmuir, 23, (2007) 452 459

8. A. Erbe, R. Sigel, Eur. Phys. J. E 22, (2007) 303-309

9. A. Stark, PhD thesis (University of Potsdam, Potsdam 2008)

10. C.F. Bohren, D.R. Huffman, Absorption and scattering of light by small particle (Wiley- $\mathrm{VCH}$, Weinheim 2004)

11. J.J. Gil, Eur. Phys. J. Appl. Phys. 40, (2007) 1-47

12. R.M.A. Azzam, N.M. Bashara, Ellipsometry and Polarized Light, Elsevier Science Publisher, Amsterdam, 1992

13. B. Lange, S.R. Aragón, J. Chem. Phys. 92, (1990) 4643-4650

14. A. Erbe, $\mathrm{PhD}$ thesis (University of Potsdam, Potsdam 2004)

15. K. Tauer, N. Weber, S. Nozari, K. Padtberg, R. Sigel, A. Stark, A. Völkel, Macromol. Symp. 281, (2009) 113

16. O. Arteaga, A. Canillas, J. Opt. Soc. Am. A 26, (2009) 783-793

17. R. Ossikovski, J. Opt. Soc. Am. A 26, (2009) 11091118 